\title{
Linear functional equations, differential operators and spectral synthesis
}

\author{
G. Kiss and M. Laczkovich
}

February 25, 2014

\begin{abstract}
We investigate the functional equation $\sum_{i=1}^{n} a_{i} f\left(b_{i} x+c_{i} y\right)=0$, where $a_{i}, b_{i}, c_{i} \in \mathbb{C}$, and the unknown function $f$ is defined on the field $K=\mathbb{Q}\left(b_{1}, \ldots, b_{n}, c_{1}, \ldots, c_{n}\right)$. (It is easy to see that every solution on $K$ can be extended to $\mathbb{C}$ as a solution.) Let $S_{1}$ denote the set of additive solutions defined on $K$. We prove that $S_{1}$ is spanned by $S_{1} \cap \mathcal{D}$, where $\mathcal{D}$ is the set of the functions $\phi \circ D$, where $\phi$ is a field automorphism of $\mathbb{C}$ and $D$ is a differential operator on $K$. We say that the equation $\sum_{i=1}^{n} a_{i} f\left(b_{i} x+c_{i} y\right)=0$ is normal, if its solutions are generalized polynomials. (The equations $\sum_{i=1}^{n} a_{i} f\left(b_{i} x+y\right)=0$ have this property.) Let $S$ denote the set of solutions of a normal equation $\sum_{i=1}^{n} a_{i} f\left(b_{i} x+c_{i} y\right)=0$ defined on $K$. We show that $S$ is spanned by $S \cap \mathcal{A}$, where $\mathcal{A}$ is the algebra generated by $\mathcal{D}$. This implies that if $S$ is translation invariant, then spectral synthesis holds in $S$. The main ingredient of the proof is the observation that if $V$ is a variety on the Abelian group $\left(K^{*}\right)^{k}$ under multiplication, and every function $F \in V$ is $k$-additive on $K^{k}$, then spectral synthesis holds in $V$.

We give several applications, and describe the set of solutions of equations having some special properties (e.g. having algebraic coefficients etc.).
\end{abstract}

\footnotetext{
${ }^{1}$ Keywords: Linear functional equations, spectral synthesis, polynomial-exponential functions

${ }^{2}$ MR subject classification: primary 43A45, 43A70, secondary $13 \mathrm{~F} 20$

${ }^{3}$ The research was partially supported by the Hungarian National Foundation for Scientific Research, Grant No. K72655 and No. K104178
} 


\section{Introduction}

Let $\mathbb{C}$ denote the field of complex numbers. We are concerned with the linear functional equation

$$
\sum_{i=1}^{n} a_{i} f\left(b_{i} x+c_{i} y\right)=0 \quad(x, y \in \mathbb{C}),
$$

where $a_{i}, b_{i}, c_{i}$ are given complex numbers, and $f: \mathbb{C} \rightarrow \mathbb{C}$ is the unknown function. We shall say that the equation (1) is normal of degree $k$, if every solution of (1) is a generalized polynomial of degree at most $k$. (For the definition of generalized polynomials see the next section.) Our aim is to describe the set of solutions of normal equations.

In fact, we shall restrict our attention to the solutions defined on the field $K$ generated by the numbers $b_{i}$ and $c_{i}(i=1, \ldots, n)$. This is justified in that any function $f: K \rightarrow \mathbb{C}$ satisfying $\sum_{i=1}^{n} a_{i} f\left(b_{i} x+\right.$ $\left.c_{i} y\right)=0$ for every $x, y \in K$ can be extended to a solution on $\mathbb{C}$. Indeed, since $\mathbb{C}$ is a linear space over the field $K$, the identity on $K$ can be extended to a function $\phi: \mathbb{C} \rightarrow K$ which is linear over $K$. It is clear that if $f$ satisfies (1) for every $x, y \in K$, then $f \circ \phi$ satisfies (1) for every $x, y \in \mathbb{C}$.

There is a simple condition on the numbers $a_{i}, b_{i}, c_{i}$ implying that the equation is normal. Suppose that the numbers $a_{i}$ are nonzero. It is well-known that the following condition implies that every solution of (1) is a generalized polynomial of degree at most $n-2$.

There is an $1 \leq i \leq n$ such that $b_{i} c_{j} \neq b_{j} c_{i}$ for any $1 \leq j \leq n, j \neq i$.

(See [7]; see also [1] and [4].) Note that condition (2) is satisfied if the numbers $b_{i}$ are different, and either $c_{i}=1$ for every $i$, or $c_{i}=1-b_{i}$ for every $i$. Therefore, the equations

$$
\sum_{i=1}^{n} a_{i} f\left(b_{i} x+y\right)=0 \quad(x, y \in \mathbb{C})
$$

and

$$
\sum_{i=1}^{n} a_{i} f\left(b_{i} x+\left(1-b_{i}\right) y\right)=0 \quad(x, y \in \mathbb{C})
$$

are normal of degree $n-2$ assuming that $a_{1}, \ldots, a_{n}$ are not all zero, and $b_{1}, \ldots, b_{n}$ are distinct. 
We shall say that the equation (1) is translation invariant, if the space of its solutions is translation invariant. It is easy to see that the equations (3) and (4) have this property. This is not true for a general equation of the form (1). As it was noted in [3], the following condition implies translation invariance:

The points $\left(b_{i}, c_{i}\right) \in \mathbb{C}^{2}$ lie on a line not going through the origin $(0,0)$.

Let $S$ denote the set of solutions defined on $K$. It is clear that $S$ is a linear space over $\mathbb{C}$ and is closed under pointwise convergence. If $S$ is also translation invariant, then $S$ is a variety. Our aim is to present a dense subset of $S$ consisting of functions of simple structure. The situation is that of spectral synthesis, and as we shall prove, spectral synthesis does hold in $S$ (see (iii) of Theorem 7.7). However, we want to present a dense subset in $S$ which is much smaller than the set of all polynomial-exponential functions, and we also want to get rid of the assumption of translation invariance. Concerning the set of additive solutions, spectral synthesis applied to $S$ itself is not informative anyway, since every additive function is a polynomial by definition. Therefore, in order to describe the additive solutions we apply spectral synthesis on the multiplicative group $K^{*}=\{x \in K: x \neq 0\}$. In general, in the case of solutions of degree $k$ we apply spectral synthesis to a related set of functions defined on $\left(K^{*}\right)^{k}$ (see Section 6).

A brief formulation of our main results is the following. Let (1) be an arbitrary equation. Then the set of additive solutions defined on $K$ is spanned by those solutions which can be written in the form $\phi \circ D$, where $\phi$ is an automorphism of $\mathbb{C}$ and $D$ is a differential operator on $K$ (see Theorem 5.1).

The set of solutions which are generalized monomials of order $k$ is spanned by those solutions which can be represented as finite sums of functions of the form $\prod_{i=1}^{k}\left(\phi_{i} \circ D_{i}\right)$, where $\phi_{1}, \ldots, \phi_{k}$ are automorphism of $\mathbb{C}$ and $D_{1}, \ldots, D_{k}$ are differential operators on $K$ (see Theorem 7.4).

If the equation (1) is normal of degree $k$, then the set $S$ is spanned by those solutions which can be can be represented as finite sums of functions of the form $\prod_{i=1}^{m}\left(\phi_{i} \circ D_{i}\right)$, where $m \leq k$, and $\phi_{i}$ and $D_{i}$ are as above (see Corollary 7.6).

The proof of these results is based on the fact that spectral synthesis holds in some related varieties (see Theorems 4.3 and 6.5). These varieties are defined on the groups $K^{*}$ and, more generally, on $\left(K^{*}\right)^{k}$. 
These groups contain free Abelian groups of rank infinity (see the remark after Theorem 3.4), and it is well-known that on such a group there are varieties in which spectral synthesis does not hold. This means that in order to prove Theorems 4.3 and 6.5 we have to use some special properties of the varieties. The crucial observation is that in these varieties every local polynomial-exponential function is a polynomial-exponential function (see Theorems 4.2 and 6.3). Then, using a general theorem stating that local spectral synthesis holds on every countable Abelian group [5] we infer that spectral synthesis holds in these varieties.

In Sections 5 and 8 we give several applications of the general theorems concerning the solutions of (1). These applications are, in a way, the continuations and completions of the groundbreaking observations, results and examples given in the papers [3] and [2].

\section{Preliminaries}

Let $K$ be a subfield of the field of complex numbers $\mathbb{C}$. A map $\phi: K \rightarrow$ $\mathbb{C}$ is an injective field homomorphism from $K$ into $\mathbb{C}$ if and only if $\phi$ is an isomorphism between the fields $K$ and $\phi(K)$. We shall frequently use the following well-known fact: if the transcendence degree of $K$ over $\mathbb{Q}$ is finite and $\phi: K \rightarrow \mathbb{C}$ is an injective field homomorphism, then $\phi$ can be extended to $\mathbb{C}$ a field automorphism of $\mathbb{C}$. In the sequel by a homomorphism (automorphism) we shall always mean a field homomorphism (a field automorphism).

Let $(G, *)$ be an Abelian group, and let $\mathbb{C}^{G}$ denote the linear space of all complex valued functions defined on $G$ equipped with the product topology. By a variety on $G$ we mean a translation invariant closed linear subspace of $\mathbb{C}^{G}$. We say that the function $f: G \rightarrow \mathbb{C}$ is additive, if $f$ is a homomorphism of $G$ into the additive group of $\mathbb{C}$. A function is a polynomial if it belongs to the algebra generated by the constant functions and the additive functions. A nonzero function $m \in \mathbb{C}^{G}$ is called an exponential if $m$ is multiplicative; that is, if $m(x * y)=m(x) \cdot m(y)$ for every $x, y \in G$. An exponential monomial is the product of a polynomial and an exponential, a polynomialexponential function is a finite sum of exponential monomials. If a variety is spanned by exponential monomials, then we say that spectral synthesis holds on this variety. If spectral synthesis holds in every variety on $G$, then we say that spectral synthesis holds on $G$. 
A function $f: G \rightarrow \mathbb{C}$ is a generalized polynomial, if there is a $k$ such that

$$
\Delta_{g_{1}} \ldots \Delta_{g_{k+1}} f=0
$$

for every $g_{1}, \ldots, g_{k+1} \in G$. Here $\Delta_{g}$ is the difference operator defined by $\Delta_{g} f(x)=f(g * x)-f(x)(x \in G)$ for every $f \in \mathbb{C}^{G}$ and $g \in G$. The smallest $k$ for which (5) holds for every $g_{1}, \ldots, g_{k+1} \in G$ is the degree of the generalized polynomial $f$.

A function $F: G^{k} \rightarrow \mathbb{C}$ is $k$-additive, if it is additive in each of its variables (the other variables being fixed). A function $f \in \mathbb{C}^{G}$ is called a generalized monomial of degree $k$, if there is a symmetric $k$-additive function $F$ such that $f(x)=F(x, \ldots, x)$ for every $x \in G$. The symmetric $k$-additive function $F$ is uniquely determined by $f$. This follows from the fact that if $F$ is symmetric, $k$-additive, and $f(x)=F(x, \ldots, x)$ for every $x \in G$, then

$$
F\left(x_{1}, \ldots, x_{k}\right)=\frac{1}{k !} \cdot \Delta_{x_{1}} \ldots \Delta_{x_{k}} f(x)
$$

for every $x_{1}, \ldots, x_{k}, x \in G$. Therefore, a function $f$ is a generalized monomial of degree $k$ if and only if the function $F$ defined by (6) is $k$-additive, and $f(x)=F(x, \ldots, x)$ for every $x \in G$.

It is well-known that every generalized polynomial of degree $k$ can be written in the form $\sum_{i=0}^{k} f_{i}$, where $f_{i}$ is a generalized monomial of degree $i$ for every $i=1, \ldots, k$, and $f_{0}$ is a constant. The following lemma is well-known (see, e.g., [5, Lemma 5]).

Lemma 2.1. Let $(G, *)$ be an Abelian group, $V$ be a translation invariant linear subspace of $\mathbb{C}^{G}$, and let $\sum_{i=1}^{M} p_{i} \cdot m_{i} \in V$, where $p_{1}, \ldots, p_{M}$ are nonzero generalized polynomials and $m_{1}, \ldots, m_{i}$ are distinct exponentials on $G$. Then $\left(\Delta_{h_{1}} \ldots \Delta_{h_{k}} p_{i}\right) \cdot m_{i} \in V$ for every $i$ and for every $h_{1}, \ldots, h_{k} \in G$. In particular, we have $m_{i} \in V$ for every $i=1, \ldots, M$.

We say that the function $f: G \rightarrow \mathbb{C}$ is a local polynomial, if, for every finitely generated subgroup $H$ of $G$, the restriction $\left.f\right|_{H}$ is a polynomial on $H$. One can prove that every polynomial is a generalized polynomial, and every generalized polynomial is a local polynomial. On finitely generated Abelian groups these notions coincide (see [5]).

The function $f: G \rightarrow \mathbb{C}$ is called a local polynomial-exponential, if $f=\sum_{i=1}^{N} p_{i} \cdot m_{i}$, where $p_{1}, \ldots, p_{N}$ are local polynomials and $m_{1}, \ldots, m_{N}$ are exponentials. Let $V$ be a variety on $G$. We say 
that local spectral synthesis holds in $V$ if the set of local polynomialexponentials contained in $V$ is dense in $V$. We say that local spectral synthesis holds on a group $G$ if local spectral synthesis holds in every variety on $G$. We shall denote by $r_{0}(G)$ the torsion free rank of $G$; that is, the cardinality of a maximal independent system of elements of infinite order. The following result was proved in [5].

Theorem 2.2. There exists a cardinal $\omega_{1} \leq \kappa \leq 2^{\omega}$ such that, for every Abelian group $G$, local spectral synthesis holds on $G$ if and only if $r_{0}(G)<\kappa$. In particular, local spectral synthesis holds on every countable Abelian group $G$.

\section{Differential operators}

For every field $K$, a derivation on $K$ is a map $d: K \rightarrow K$ such that $d(x+y)=d(x)+d(y)$ and $d(x y)=d(x) \cdot y+d(y) \cdot x$ for every $x, y \in K$. It is well-known that if $d$ is a derivation on $K$ and $L$ is a field containing $K$, then $d$ can be extended to $L$ as a derivation.

Suppose that the complex numbers $t_{1}, \ldots, t_{n}$ are algebraically independent over $\mathbb{Q}$. By a differential operator on $\mathbb{Q}\left(t_{1}, \ldots, t_{n}\right)$ we mean an operator of the form

$$
D=\sum c_{i_{1}, \ldots, i_{n}} \cdot \frac{\partial^{i_{1}+\cdots+i_{n}}}{\partial t_{1}^{i_{1}} \cdots \partial t_{n}^{i_{n}}},
$$

where the sum is finite, in each term the coefficient is a complex number, and the exponents $i_{1}, \ldots, i_{n}$ are nonnegative integers. The degree of the differential operator $D$ is the maximum of the numbers $i_{1}+\ldots+i_{n}$ such that $c_{i_{1}, \ldots, i_{n}} \neq 0$.

It is obvious that $\partial / \partial t_{i}$ is a derivation on $\mathbb{Q}\left(t_{1}, \ldots, t_{n}\right)$ for every $i=1, \ldots, n$. Therefore, every differential operator on $\mathbb{Q}\left(t_{1}, \ldots, t_{n}\right)$ is the linear combination with complex coefficients of finitely many maps of the form $d_{1} \circ \ldots \circ d_{k}$, where $d_{1}, \ldots, d_{k}$ are derivations on $\mathbb{Q}\left(t_{1}, \ldots, t_{n}\right)$. This observation motivates the following definition.

Definition 3.1. Let $K$ be a subfield of $\mathbb{C}$. We say that the map $D$ : $K \rightarrow \mathbb{C}$ is a differential operator on $K$, if $D$ is the linear combination, with complex coefficients, of finitely many maps of the form $d_{1} \circ \ldots \circ d_{k}$, where $d_{1}, \ldots, d_{k}$ are derivations on $K$.

Note that if $K \subset L \subset \mathbb{C}$ are fields and $D$ is a differential operator on $K$, then $D$ can be extended to $L$ as a differential operator. This is 
clear from the fact that every derivation can be extended from $K$ to $L$.

We show that if $K=\mathbb{Q}\left(t_{1}, \ldots, t_{n}\right)$, then the two definitions of differential operators coincide. Actually, more is true.

Proposition 3.2. Let $K$ be a subfield of $\mathbb{C}$, and suppose that the elements $t_{1}, \ldots, t_{n} \in K$ are algebraically independent over $\mathbb{Q}$. If $D$ is a differential operator on $K$ according to Definition 3.1, then the restriction of $D$ to $\mathbb{Q}\left(t_{1}, \ldots, t_{n}\right)$ is of the form $(7)$.

Proof. Put $\mathbb{Q}\left(t_{1}, \ldots, t_{n}\right)=F$, and let $\mathcal{D}$ denote the set of all functions defined on $F$ that can be represented in the form (7). It is enough to show that if $d_{1}, \ldots, d_{k}$ are derivations on $K$, then the restriction of $d_{1} \circ \ldots \circ d_{k}$ to $F$ belongs to $\mathcal{D}$. We prove this by induction on $k$. First we note that if $d$ is a derivation on $K$ and $d\left(t_{i}\right)=\alpha_{i}(i=1, \ldots, n)$, then

$$
d(x)=\sum_{i=1}^{n} \alpha_{i} \cdot \frac{\partial x}{\partial t_{i}}
$$

for every $x \in F$. Indeed, (8) can be easily checked first for every $x \in \mathbb{Q}\left[t_{1}, \ldots, t_{n}\right]$ and then for every $x \in F$. Therefore, the statement is true for $k=1$.

Let $k>1$, and suppose the statement is true for $k-1$. Let $d_{1}, \ldots, d_{k}$ be derivations on $K$. Then, by the induction hypothesis, the map $g=d_{2} \circ \ldots \circ d_{k}$ restricted to $F$ belongs to $\mathcal{D}$. Let $g=\sum_{j=1}^{N} c_{j} g_{j}$, where each $g_{j}$ is of the form $\partial^{i_{1}+\ldots+i_{n}} / \partial t_{1}^{i_{1}} \cdots \partial t_{n}^{i_{n}}$. Extend $d_{1}$ to $\mathbb{C}$ as a derivation. Then

$$
d_{1} \circ g=d_{1} \circ\left(\sum_{j=1}^{N} c_{j} g_{j}\right)=\sum_{j=1}^{N}\left(d_{1}\left(c_{j}\right) \cdot g_{j}+c_{j} \cdot\left(d_{1} \circ g_{j}\right)\right) .
$$

Now the statement $\left.\left(d_{1} \circ g\right)\right|_{F} \in \mathcal{D}$ follows from (8) when applied to $d=d_{1}$. $\mathbb{C}$.

In the sequel we shall denote by $j$ the identity function defined on

Theorem 3.3. Let $K$ be a subfield of $\mathbb{C}$, and let $D$ be a differential operator on $K$. Then $D / j$ is a polynomial on $K^{*}$.

Proof. It is enough to show that if $d_{1}, \ldots, d_{n}$ are derivations on $K$, then $\left(d_{1} \circ \ldots \circ d_{n}\right) / j$ is a polynomial on $K^{*}$. We prove by induction 
on $n$. It is easy to check that if $d$ is a derivation, then $d / j$ is additive on $K$. Since every additive function is a polynomial, the statement is true for $n=1$.

Suppose that $n>1$, and the statement is true for $n-1$. Let $d_{1}, \ldots, d_{n}$ be derivations on $K$. By the induction hypothesis, $\left(d_{2} \circ\right.$ $\left.\ldots \circ d_{n}\right) / j=p$ is a polynomial on $K^{*}$. Extend $p$ to $K$ by putting $p(0)=0$. We have to show that $\left(d_{1} \circ(p \cdot j)\right) / j$ is a polynomial on $K^{*}$. Since $d_{1}$ is a derivation, we have

$$
d_{1}(p(x) \cdot x)=d_{1}(p(x)) \cdot x+p(x) \cdot d_{1}(x)
$$

for every $x \in K$. Thus $\left(d_{1} \circ(p \cdot j)\right) / j=\left(d_{1} \circ p\right)+p \cdot\left(d_{1} / j\right)$ on $K^{*}$. Since $p$ is a polynomial and $d_{1} / j$ is additive on $K^{*}$, it follows that $p \cdot\left(d_{1} / j\right)$ is a polynomial on $K^{*}$. Therefore, it is enough to show that $d_{1} \circ p$ is a polynomial on $K^{*}$.

Extend $d_{1}$ to $\mathbb{C}$ as a derivation. Let $d_{1} / j=a$; then $a$ is additive on $\mathbb{C}^{*}$, and $d_{1}=a \cdot j$, where we extended $a$ to $\mathbb{C}$ by putting $a(0)=0$. (The additivity of $a$ on $\mathbb{C}^{*}$ means $a(x y)=a(x)+a(y)$ for every $x, y \in$ $\mathbb{C}^{*}$.) Now $p$ is a sum of functions of the form $a_{1} \cdots a_{k}$, where each of $a_{1}, \ldots, a_{k}$ is either additive on $K^{*}$ or constant. Since $d_{1}$ is additive on $\mathbb{C}$, it is enough to show that $d_{1} \circ\left(a_{1} \cdots a_{k}\right)$ is a polynomial on $K^{*}$. We have

$$
\begin{aligned}
d_{1} \circ\left(a_{1} \cdots a_{k}\right) & =(a \cdot j) \circ\left(a_{1} \cdots a_{k}\right)=\left(a \circ\left(a_{1} \cdots a_{k}\right)\right) \cdot a_{1} \cdots a_{k}= \\
& =\left[\left(a \circ a_{1}\right)+\ldots+\left(a \circ a_{k}\right)\right] \cdot a_{1} \cdots a_{k}
\end{aligned}
$$

everywhere on $K$. Since $a \circ a_{i}$ is either constant or additive on $K^{*}$, it follows that the right hand side of (9) is a polynomial on $K^{*}$.

Our next aim is to prove the following result.

Theorem 3.4. Suppose that the transcendence degree of the field $K$ over $\mathbb{Q}$ is finite, and let the map $D: K \rightarrow \mathbb{C}$ be additive. Then the following are equivalent.

(i) $D$ is a differential operator on $K$.

(ii) $D / j$ is a polynomial on $K^{*}$.

(iii) $D / j$ is a generalized polynomial on $K^{*}$.

(iv) $D / j$ is a local polynomial on $K^{*}$. 
We remark that the torsion free rank of the Abelian group $K^{*}$ is infinite for any $K$. Indeed, the set of rational primes constitutes an independent family of elements of infinite order in $K^{*}$. Therefore, for any field $K \subset \mathbb{C}$, the families of polynomials, generalized polynomials, and local polynomials defined on $K^{*}$ are different.

In the next two lemmas we shall use the following notation. Let $K$ be a field as in Theorem 3.4, and let $T \subset K$ be a maximal set of algebraically independent elements over $\mathbb{Q}$. By assumption, $T$ is finite; let $T=\left\{t_{1}, \ldots, t_{n}\right\}$. We shall denote by $G$ the subgroup of $K^{*}$ generated by $t_{1}, \ldots, t_{n}$. Then $G$ is a finitely generated subgroup of $K^{*}$.

Lemma 3.5. Let $f: K \rightarrow \mathbb{C}$ be an additive function. Let $H$ be a subgroup of $K^{*}$ such that $G \subset H \subset K^{*}$. Suppose that $p=f / j$ is a generalized polynomial on $H$. If $f \equiv 0$ on $G$, then $f \equiv 0$ on $H$.

Proof. We prove by induction on the degree of the generalized polynomial $p$ on $H$. If $\operatorname{deg} p=0$, then $p$ is constant. Since $p \equiv 0$ on $G$, we have $p \equiv 0$ on $H$, and $f \equiv 0$ on $H$.

Suppose $m=\operatorname{deg} p>0$, and that the statement is true for degrees less than $m$. Let $g \in G$ be fixed. Then

$$
p(g x)-p(x)=\frac{f(g x)}{g x}-\frac{f(x)}{x}=\frac{g^{-1} f(g x)-f(x)}{x}=\frac{f_{1}(x)}{x},
$$

where $f_{1}(x)=g^{-1} f(g x)-f(x)$ for every $x \in K$. Then $f_{1}$ is additive on $K$, and $f_{1} / j$ is a generalized polynomial on $H$ by (10). Moreover, we have $f_{1} / j=\Delta_{g} p$, and thus $\operatorname{deg}\left(\left.\left(f_{1} / j\right)\right|_{H}\right) \leq m-1$. Since $f_{1} \equiv 0$ on $G$, it follows from the induction hypothesis that $f_{1} \equiv 0$ on $H$. Thus $f(g x)=g \cdot f(x)$ for every $g \in G$ and $x \in H$. By the additivity of $f$ we obtain

$$
f(c x)=c \cdot f(x) \quad(c \in \mathbb{Q}[T], x \in H) .
$$

Let $\alpha \in H$ be arbitrary. Then, by $\alpha \in K, \alpha$ is algebraic over the field $\mathbb{Q}(T)$. Let $c_{0}, \ldots, c_{k} \in \mathbb{Q}[T]$ be such that

$$
c_{k} \alpha^{k}+\ldots+c_{1} \alpha+c_{0}=0,
$$

$c_{k} \neq 0$ and $k$ is minimal. Let $f\left(\alpha^{i}\right)=a_{i}(i \in \mathbb{Z})$. Multiplying (12) by $\alpha^{n-k}$ for every $n \in \mathbb{Z}$ we obtain

$$
c_{k} \alpha^{n}+\ldots+c_{1} \alpha^{n-k+1}+c_{0} \alpha^{n-k}=0 .
$$


By (11) and by the additivity of $f$, this implies

$$
c_{k} a_{n}+\ldots+c_{1} a_{n-k+1}+c_{0} a_{n-k}=0
$$

for every $n$. Therefore, the sequence $\left(a_{n}\right)$ satisfies a linear recurrence relation. It is well-known that $a_{n}$ can be uniquely represented in the form $a_{n}=\sum_{\lambda \in \Lambda} q_{\lambda}(n) \cdot \lambda^{n}$, where $\lambda$ runs through $\Lambda$, the set of roots of the characteristic polynomial $\chi(x)=c_{k} x^{k}+\ldots+c_{0}$, and for every root $\lambda \in \Lambda, q_{\lambda} \in \mathbb{C}[x]$ is a polynomial of the degree less than the multiplicity of $\lambda$.

By the minimality of $k$, the polynomial $\chi$ is irreducible over $\mathbb{Q}(T)$. Therefore, every $\lambda$ is a simple root of $\chi$, and thus

$$
a_{n}=\sum_{\lambda \in \Lambda} d_{\lambda} \cdot \lambda^{n}
$$

for every $n$, where $d_{\lambda}$ is a constant for every $\lambda \in \Lambda$.

Since $p$ is a generalized polynomial on $H$ and $\left\{\alpha^{n}\right\}$ is a finitely generated subgroup of $H$, it follows that $p$ is a polynomial on $\left\{\alpha^{n}\right\}$ (see [4]). Therefore, the map $n \mapsto p\left(\alpha^{n}\right)(n \in \mathbb{Z})$ is a polynomial on $\mathbb{Z}$. Now, we have $a_{n}=f\left(\alpha^{n}\right)=p\left(\alpha^{n}\right) \cdot \alpha^{n}$ for every $n$. The uniqueness of the representation (13) implies that $\alpha \in \Lambda$ and the function $n \mapsto p\left(\alpha^{n}\right)(n \in \mathbb{Z})$ is constant. Since $p(1)=f(1)=0$ by $1 \in G$, it follows that $p\left(\alpha^{n}\right)=0$ for every $n$. In particular, $p(\alpha)=0$ and $f(\alpha)=0$. Since this is true for every $\alpha \in H$, we obtain $f \equiv 0$ on $H$.

Lemma 3.6. Let $f: K \rightarrow \mathbb{C}$ be an additive function such that $p=f / j$ is a local polynomial on $K^{*}$. If $f \equiv 0$ on $G$, then $f \equiv 0$ on $K$.

Proof. The additivity of $f$ implies $f(0)=0$. Let $\alpha \in K^{*}$ be arbitrary, and let $H$ be the multiplicative group generated by $T$ and $\alpha$. Since $H$ is a finitely generated subgroup of $K^{*}$, it follows that $p$ is a polynomial on $H$. By the previous lemma we obtain that $f \equiv 0$ on $H$. In particular, $f(\alpha)=0$. Since this is true for every $\alpha \in K^{*}$, we obtain $f \equiv 0$ on $K^{*}$.

Proof of Theorem 3.4. The implication (i) $\Longrightarrow$ (ii) was proved in Theorem 3.3 (for every field). The implications (ii) $\Longrightarrow$ (iii) $\Longrightarrow$ (iv) are obvious.

Now we prove (iv) $\Longrightarrow(\mathrm{i})$. Let $p=D / j$, then $p$ is a local polynomial on $K^{*}$. Since $G$ is a finitely generated subgroup of $K^{*}$, it follows that 
$p$ is a polynomial on $G$. By [4, Proposition 1], $p$ has the $P_{l o c}$ property on $G$; that is, the map

$$
\left(k_{1}, \ldots, k_{n}\right) \mapsto p\left(t_{1}^{k_{1}} \cdots t_{n}^{k_{n}}\right)
$$

is a polynomial on $\mathbb{Z}^{n}$.

We shall use the notation $x^{[0]}=1$ and $x^{[i]}=x(x-1) \cdots(x-$ $i+1)$ for every $i=1,2, \ldots$ and $x \in \mathbb{Z}$. It is well-known that every polynomial belonging to $\mathbb{C}\left[x_{1}, \ldots, x_{n}\right]$ can be written in the form $\sum c_{i}$. $x_{1}^{\left[i_{1}\right]} \cdots x_{n}^{\left[i_{n}\right]}$, where $i=\left(i_{1}, \ldots, i_{n}\right)$ runs through a finite set of $n$-tuples of nonnegative integers, and in each term the coefficient $c_{i}$ is a complex number. Therefore, the map $\left(k_{1}, \ldots, k_{n}\right) \mapsto p\left(t_{1}^{k_{1}} \cdots t_{n}^{k_{n}}\right)$ has such a representation. Then we have

$$
\begin{aligned}
D\left(t_{1}^{k_{1}} \cdots t_{n}^{k_{n}}\right) & =p\left(t_{1}^{k_{1}} \cdots t_{n}^{k_{n}}\right) \cdot t_{1}^{k_{1}} \cdots t_{n}^{k_{n}}= \\
& =\sum c_{i} \cdot k_{1}^{\left[i_{1}\right]} \cdots k_{n}^{\left[i_{n}\right]} \cdot t_{1}^{k_{1}} \cdots t_{n}^{k_{n}}= \\
& =\sum c_{i} \cdot t_{1}^{i_{1}} \cdots t_{n}^{i_{n}} \cdot k_{1}^{\left[i_{1}\right]} \cdots k_{n}^{\left[i_{n}\right]} \cdot t_{1}^{k_{1}-i_{1}} \cdots t_{n}^{k_{n}-i_{n}}= \\
& =E\left(t_{1}^{k_{1}} \cdots t_{n}^{k_{n}}\right)
\end{aligned}
$$

for every $k_{1}, \ldots, k_{n} \in \mathbb{Z}$, where $E$ is the differential operator

$$
\sum c_{i} \cdot t_{1}^{i_{1}} \cdots t_{n}^{i_{n}} \cdot \frac{\partial^{i_{1}+\cdots+i_{n}}}{\partial t_{1}^{i_{1}} \cdots \partial t_{n}^{i_{n}}} .
$$

By extending the derivations $\partial / \partial t_{i}$ to $K$, we can extend $E$ to $K$ as a differential operator $\bar{E}$. Then $\bar{E}$ is additive on $K$, and $\bar{E} / j$ is a polynomial on $K^{*}$ by Theorem 3.3. Let $q(0)=0$, and let $q(x)=$ $p(x)-\bar{E}(x) / x$ for every $x \in K^{*}$. Then $q \cdot j=D-\bar{E}$ is additive on $K$, and $q$ is a local polynomial on $K^{*}$. Since $q$ vanishes on $G$ by (14), it follows from Lemma 3.6 that $q \equiv 0$ on $K$. Thus $D=\bar{E}$ on $K$ which completes the proof.

\section{Spectral synthesis on $K^{*}$ in the va- riety of additive functions on $K$}

Let $K$ be a subfield of $\mathbb{C}$, and let $K^{*}$ denote the Abelian group $\{x \in$ $K: x \neq 0\}$ with respect to multiplication. 
In this section we fix a field $K$ such that its transcendence degree over $\mathbb{Q}$ is finite. The function $f: K \rightarrow \mathbb{C}$ is said to be additive, if $f(x+y)=f(x)+f(y)$ holds for every $x, y \in K$. We denote by $V_{a}$ the set of additive functions $f: K \rightarrow \mathbb{C}$.

We put $V_{a}^{*}=\left\{\left.f\right|_{K^{*}}: f \in V_{a}\right\}$. It is easy to check that $V_{a}^{*}$ is a variety on $K^{*}$ (see the proof of [2, Theorem 2.3]).

Our aim is to prove that spectral synthesis holds in every variety on $K^{*}$ contained by $V_{a}$ (see Theorem 4.3). It should be remarked that, as the torsion free rank of $K^{*}$ is infinite (see the remark after Theorem 3.4), there are varieties on $K^{*}$ in which spectral synthesis does not hold (see [8] and [6]).

Lemma 4.1. If $m \in V_{a}^{*}$ is an exponential on $K^{*}$, then $m$ can be extended to $\mathbb{C}$ as an automorphism of $\mathbb{C}$.

Proof. The condition $m \in V_{a}^{*}$ means that extending $m$ to $K$ by $m(0)=0$, we obtain an additive function. Now $m$ is an exponential on $K^{*}$, and thus $m$ satisfies $m(x y)=m(x) m(y)$ for every $x, y \in K^{*}$. Consequently, the extended $m$ is an injective homomorphism of $K$. Since the transcendence degree of $K$ over $\mathbb{Q}$ is finite, it follows that $m$ can be extended to $\mathbb{C}$ as an automorphism of $\mathbb{C}$.

Theorem 4.2. Suppose that the transcendence degree of the field $K$ over $\mathbb{Q}$ is finite. Let $f: K \rightarrow \mathbb{C}$ be additive, and let $m$ be an exponential on $K^{*}$. Let $\phi$ be an extension of $m$ to $\mathbb{C}$ as an automorphism of

$\mathbb{C}$. Then the following are equivalent.

(i) $f=p \cdot m$ on $K^{*}$, where $p$ is a local polynomial on $K^{*}$.

(ii) $f=p \cdot m$ on $K^{*}$, where $p$ is a generalized polynomial on $K^{*}$.

(iii) $f=p \cdot m$ on $K^{*}$, where $p$ is a polynomial on $K^{*}$.

(iv) There exists a unique differential operator $D$ on $K$ such that $f=\phi \circ D$ on $K$.

Proof. The implications (iii) $\Longrightarrow($ ii $) \Longrightarrow$ (i) are obvious.

(i) $\Longrightarrow\left(\right.$ iv): Let $D=\phi^{-1} \circ f$. Then $D$ is additive on $K$, and

$$
D=\phi^{-1} \circ(p \cdot m)=\left(\phi^{-1} \circ p\right) \cdot j
$$

on $K$. Thus $D / j=\phi^{-1} \circ p$ on $K^{*}$. Since $p$ is a local polynomial on $K^{*}$ and $\phi^{-1}$ is an automorphism of $\mathbb{C}$, it follows that $\phi^{-1} \circ p=D / j$ is a local polynomial on $K^{*}$. Therefore, $D$ is a differential operator on 
$K$ by Theorem 3.4. Since $f=\phi \circ D$ on $K$, this proves the existence of $D$. The uniqueness is clear: if $\phi \circ D_{1}=\phi \circ D_{2}$ on $K$, then $D_{1}=D_{2}$, since $\phi$ is injective.

(iv) $\Longrightarrow$ (iii): Suppose $f=\phi \circ D$ on $K$, where $D$ is a differential operator on $K$. Then $D / j$ is a polynomial on $K^{*}$, and so is

$$
p=\phi \circ(D / j)=(\phi \circ D) / \phi .
$$

Thus $f=\phi \circ D=p \cdot \phi$. Since $m=\phi$ on $K$, the proof is complete.

Theorem 4.3. Suppose that the transcendence degree of the field $K$ over $\mathbb{Q}$ is finite. Then spectral synthesis holds in every variety on $K^{*}$ consisting of additive functions (with respect to addition).

Proof. Since $K$ is countable, so is the Abelian group $K^{*}$. Let $V$ be a variety on $K^{*}$ consisting of additive functions. By Theorem 2.2, local spectral synthesis holds on $K^{*}$, and thus $V$ is spanned by local polynomial-exponential functions. Since, by Theorem 4.2, every local polynomial-exponential function contained by $V$ is a polynomialexponential function, it follows that $V$ is spanned by polynomialexponential functions.

\section{Applications to linear functional equa- tions}

As an application of Theorems 4.2 and 4.3 we describe the additive solutions of the linear functional equation

$$
\sum_{i=1}^{n} a_{i} f\left(b_{i} x+c_{i} y\right)=0,
$$

where $a_{i}, b_{i}, c_{i}$ are given complex numbers and $f: \mathbb{C} \rightarrow \mathbb{C}$ is the unknown function. Let $K=\mathbb{Q}\left(b_{1}, \ldots, b_{n}, c_{1}, \ldots, c_{n}\right)$. Let $S_{1}$ denote the set of additive solutions of (15) defined on $K$. Clearly, $f: K \rightarrow \mathbb{C}$ belongs to $S_{1}$ if and only if

$$
\sum_{i=1}^{n} a_{i} f\left(b_{i} x\right)=0, \quad \sum_{i=1}^{n} a_{i} f\left(c_{i} x\right)=0
$$

holds for every $x \in K$. It is also clear that $S_{1}$ is a linear space over $\mathbb{C}$. It is easy to check that

$$
S_{1}^{*}=\left\{\left.f\right|_{K^{*}}: f \in S_{1}\right\}
$$


is a variety on $K^{*}$.

The next theorem is our main result concerning the additive solutions of linear functional equations.

Theorem 5.1. (i) For every function $f \in S_{1}^{*}, f$ is an exponential monomial on $K^{*}$ if and only if $f=\phi \circ D$ on $K^{*}$, where $\phi$ is an automorphism of $\mathbb{C}$ and is a solution of (15), and $D$ is a differential operator on $K$.

(ii) The variety $S_{1}^{*}$ is spanned by the functions $\left.(\phi \circ D)\right|_{K^{*}} \in S_{1}^{*}$, where $\phi$ and $D$ are as above.

(iii) The linear space $S_{1}$ is spanned by the functions $\phi \circ D$, where $\phi$ and $D$ are as above.

Proof. (i) Suppose that $f: K \rightarrow \mathbb{C}$ is an additive solution of (15), and $f$ is an exponential monomial function on $K^{*}$. Let $f=p \cdot m$, where $p$ is a polynomial, and $m$ is an exponential on $K^{*}$. Since $V_{a}^{*}$ is a variety and $p \cdot m \in V_{a}^{*}$, it follows that $m \in V_{a}^{*}$ (see Lemma 2.1). This means that defining $m(0)=0$, the function $m$ is a solution of (15) on $K$. By Lemma 4.1, $m$ can be extended as an automorphism of $\mathbb{C}$. Let $\phi$ denote such an extension. As $m$ is a solution of (16) as well, we have

$$
\sum_{i=1}^{n} a_{i} m\left(b_{i}\right)=0, \quad \sum_{i=1}^{n} a_{i} m\left(c_{i}\right)=0 .
$$

Then, by (17), $\phi$ is a solution of $(15)$ on $\mathbb{C}$. The rest of the statement (i) follows from Theorem 4.2.

Statement (ii) is an immediate consequence of Theorem 4.3. The statement (iii) is clear from (ii).

The description of the additive solutions becomes especially simple if the coefficients $a_{i}$ are algebraic.

Theorem 5.2. Suppose that $a_{1}, \ldots, a_{n}$ are algebraic numbers. If $\phi$ is an automorphism of $\mathbb{C}$ and $\phi$ is a solution of $(15)$, then $\phi \circ D \in S_{1}$ for every differential operator $D$ on $K$. Therefore, $S_{1}^{*}$ is spanned by the functions $\left.(\phi \circ D)\right|_{K^{*}} \in S_{1}^{*}$, where $\phi$ is an automorphism of $\mathbb{C}$ and is a solution of (15), and $D$ is an arbitrary differential operator on $K$.

Proof. Since we are only interested in the additive solutions of (15), it is enough to deal with the additive solutions of the system (16). 
It is enough to show that $\phi \circ D$ is a solution of (16) on $K$ for any differential operator $D=d_{1} \circ \cdots \circ d_{k}$, where $d_{1}, \ldots, d_{k}$ are derivations. We will prove this by induction on $k$.

If $k=0$; that is, if $D$ is the identity, then $\phi \circ D=\phi$ is a solution by assumption.

Let $k>0$, and suppose the statement is true for $k-1$. We have to prove that if $d_{1}, \ldots, d_{k}$ are derivations on $K$, then $\phi \circ\left(d_{1} \circ \cdots \circ d_{k}\right)$ is a solution on $K$. We have

$$
\phi \circ\left(d_{1} \circ \cdots \circ d_{k}\right)=d \circ f
$$

where $d=\phi \circ d_{1} \circ \phi^{-1}$ and $f=\phi \circ\left(d_{2} \circ \cdots \circ d_{k}\right)$. Then $f: K \rightarrow \phi(K)$ is a solution of (16) by the induction hypothesis.

Let $K_{1}=K\left(\phi^{-1}\left(a_{1}\right), \ldots, \phi^{-1}\left(a_{n}\right)\right)$, and let $d_{1}$ be extended to $K_{1}$ as a derivation. We denote the extended derivation by $\bar{d}_{1}$. Note that $\bar{d}_{1}(a)=0$ for every algebraic element of $K_{1}$.

Let $\bar{d}=\phi \circ \bar{d}_{1} \circ \phi^{-1}$. It is easy to check that $\bar{d}$ is a derivation on $\phi\left(K_{1}\right)$. If $a \in \phi\left(K_{1}\right)$ is algebraic, then so is $\phi^{-1}(a)$, and thus $\bar{d}_{1}\left(\phi^{-1}(a)\right)=0$. Therefore, $\bar{d}(a)=0$ for every algebraic element of $\phi\left(K_{1}\right)$. In particular, $\bar{d}\left(a_{i}\right)=0$ for every $i=1, \ldots, n$. Since $f$ is a solution of (16) we have, for every $x \in K$,

$$
\begin{aligned}
0 & \left.=\bar{d}(0)=\bar{d}\left(\sum_{i=1}^{n} a_{i} \cdot f\left(b_{i} x\right)\right)=\sum_{i=1}^{n} \bar{d}\left(a_{i} \cdot f\left(b_{i} x\right)\right)\right)= \\
& =\sum_{i=1}^{n} \bar{d}\left(a_{i}\right) \cdot f\left(b_{i} x\right)+\sum_{i=1}^{n} a_{i} \cdot d\left(f\left(b_{i} x\right)\right)=\sum_{i=1}^{n} a_{i} \cdot(d \circ f)\left(b_{i} x\right) .
\end{aligned}
$$

The same argument shows that $\sum_{i=1}^{n} a_{i} \cdot(d \circ f)\left(c_{i} x\right)=0$ for every $x \in K$. Thus $\phi \circ d$ is a solution of (16) on $K$ which completes the proof.

By Theorems 5.1 and 5.2 we have the following corollary.

Corollary 5.3. If the coefficients $a_{1}, \ldots, a_{n}$ are algebraic, then the variety of additive solutions of (15) defined on $K$ is spanned by the functions $\phi \circ D$, where $\phi$ is an isomorphism solution and $D$ is an arbitrary differential operator.

Theorem 5.4. Suppose that $a_{1}, \ldots, a_{n}$ are algebraic and that (15) has a nonzero additive solution on $K$. Then $S_{1}$ is of finite dimension over $\mathbb{C}$ if and only if each of the numbers $b_{1}, \ldots, b_{n}, c_{1}, \ldots, c_{n}$ is algebraic. 
Proof. If $b_{i}$ and $c_{i}$ are algebraic numbers, then the field

$$
K=\mathbb{Q}\left(b_{1}, \ldots, b_{n}, c_{1}, \ldots, c_{n}\right)
$$

is a finite dimensional linear space over $\mathbb{Q}$. Consequently, the linear space of additive functions defined on $K$ is also of finite dimension. This implies that the space of additive solutions of (15) is, a fortiori, of finite dimension.

Next suppose that at least one of the numbers $b_{1}, \ldots, b_{n}, c_{1}, \ldots, c_{n}$ is transcendental. We show that if (15) has a nonzero additive solution on $K$, then the set $S_{1}$ of all nonzero additive solution defined on $K$ has infinite dimension over $\mathbb{Q}$.

By [3, Theorem 2.3] (or, by (iii) of Theorem 5.1), there is an automorphism $\phi$ of $\mathbb{C}$ which is a solution of (15) on $K$. Let $T$ be a maximal subset of $K$ consisting of algebraically independent elements over $\mathbb{Q}$. Since the degree of transcendence of $K$ is at least $1, T \neq \emptyset$. Let $t \in T$ be selected. For every $n$ there is a differential operator $D_{n}$ on $K$ which is an extension of $\frac{\partial^{n}}{\partial t^{n}}$ from $\mathbb{Q}(T)$. Clearly, the operators $D_{n}$ are linearly independent over $\mathbb{Q}(T)$. Then so are the maps $\phi \circ D_{n}$. Since, by Theorem 5.2, the maps $\phi \circ D_{n}$ are additive solutions of (15) on $K$, the proof is complete.

If $b_{i}$ and $c_{i}$ are algebraic numbers, then every differential operator on $K$ is a constant multiple of the identity. Therefore, in this case a finite basis of the linear space $S_{1}$ consists of the injective homomorphisms satisfying

$$
\sum_{i=1}^{n} a_{i} \phi_{j}\left(b_{i}\right)=0 \text { and } \sum_{i=1}^{n} a_{i} \phi_{j}\left(c_{i}\right)=0
$$

for every $j \in\{1, \ldots, k\}$. See also [2, Theorem 2.3].

The following example shows that if the numbers $b_{i}$ and $c_{i}$ are not all algebraic, then the injective homomorphism solutions do not necessarily span $S_{1}$; that is, we may need nontrivial differential operators in order to generate $S_{1}$. Consider the functional equation

$$
f\left(t^{2} x+y\right)-2 t f(t x+y)+t^{2} f(x+y)-(t-1)^{2} f(y)=0,
$$

where $t$ is a fixed transcendental number. Then $K=\mathbb{Q}(t)$. If $\phi$ is an injective homomorphism solution on $K$ then

$$
\phi\left(t^{2}\right)-2 t \phi(t)+t^{2} \phi(1)=0 .
$$


Then we have $\phi(t)=t$, and thus $\phi$ is the identity on $K$. Now, $\phi$ does not generate $S_{1}$. Indeed, an easy computation shows that the differential operator $\frac{\partial}{\partial t}$ is a solution of (19) on $K$. Since $\frac{\partial}{\partial t}$ is not a constant multiple of the identity, $S_{1}$ is not spanned by (the unique) injective homomorphism solution.

We remark that $\frac{\partial}{\partial t^{k}}$ is not a solution of (19) if $k \geq 2$, and thus $S_{1}$ is of finite dimension over $\mathbb{Q}$. In the next theorem we show that this behaviour is typical, supposing that $b_{i}, c_{i}$ generate a purely transcendental field of transcendence degree 1.

Definition 5.5. An equation of the form (15) is called trivial if every additive function $f: \mathbb{C} \rightarrow \mathbb{C}$ is a solution.

Theorem 5.6. Suppose that $b_{1}, \ldots, b_{n}, c_{1}, \ldots, c_{n} \in \mathbb{Q}(t)$, where $t$ is transcendental over $\mathbb{Q}$. Then the equation (15) is either trivial or $S_{1}$ is of finite dimension over $\mathbb{C}$.

Proof. The additive solutions of (15) are the same as the solutions of the system (16). If both of the equations of (16) are trivial, then so is (15). Also, if the space of additive solutions of any of the equations of (16) is of finite dimension, then the same is true for the space of additive solutions of (15). Therefore, it is enough to show that if the equation $\sum_{i=1}^{n} a_{i} f\left(b_{i} x\right)=0$ is nontrivial, then the linear space of its additive solutions defined on $\mathbb{Q}(t)$ is of finite dimension.

For every $\gamma \neq 0$, the equations $\sum_{i=1}^{n} a_{i} f\left(b_{i} x\right)=0$ and

$$
\sum_{i=1}^{n} a_{i} f\left(b_{i} \gamma x\right)=0
$$

are equivalent in the sense that if one of the equations is trivial then so is the other, and if the space of additive solutions of one of them is of finite dimension, then the same is true for the other.

By assumption, $b_{1}, \ldots, b_{n}$ are rational functions of $t$ with rational coefficients. Let $\gamma$ denote the common denominator of $b_{1}, \ldots, b_{n}$. Then $b_{i} \gamma \in \mathbb{Q}[t]$. Let $b_{i} \gamma=\sum_{j=0}^{m} \alpha_{i, j} t^{j}$, where $\alpha_{i, j} \in \mathbb{Q}$ for every $i, j$. Since we are interested in the additive solutions only, we may replace each term $f\left(b_{i} \gamma x\right)$ by $\sum \alpha_{i, j} f\left(t^{j} x\right)$ (here we used the fact that $f(\alpha x)=$ $\alpha \cdot f(x)$ for every rational $\alpha)$. Collecting the terms $a_{i} \cdot \alpha_{i, j} f\left(t^{j} x\right)$ in the sum $\sum_{i=1}^{n} a_{i} f\left(b_{i} \gamma x\right)$ for every $j$, we find that there is an equation

$$
\sum_{j=0}^{m} A_{j} f\left(t^{j} x\right)=0
$$


such that the additive solutions of (20) and those of (21) coincide. If $A_{j}=0$ for every $j=1, \ldots, m$, then the equations are trivial. Therefore, we may assume that $A_{j} \neq 0$ for some $j$. We prove that in this case the set of additive solutions of $(21)$ defined on $\mathbb{Q}(t)$ is of finite dimension.

Let $\Phi$ denote the set of functions $\phi \circ D$, where $\phi$ is an injective homomorphism of $\mathbb{Q}(t)$ and is a solution of $(21), D$ is a differential operator of $\mathbb{Q}(t)$, and $\phi \circ D$ is a solution of (21). By Theorem 5.1, applied to the equation $\sum_{j=0}^{m} A_{j} f\left(t^{j} x+0 \cdot y\right)=0$, we find that the linear space of additive solutions of (21) is spanned by $\Phi$ and thus it is enough to show that $\Phi$ generates a finite dimensional linear space over $\mathbb{C}$.

If $\phi$ is an injective homomorphism of $\mathbb{Q}(t)$ and a solution of $(21)$, then we have

$$
\sum_{j=0}^{m} A_{j} \phi\left(t^{j} x\right)=0
$$

for every $x \in \mathbb{Q}(t)$. Putting $x=1$ and using $\phi\left(t^{j}\right)=(\phi(t))^{j}$ we find that $\phi(t)$ is a root of the polynomial $P(X)=\sum_{j=0}^{m} A_{j} \cdot X^{j}$. Since $P$ only has a finite number of roots and $\phi$ is determined by the value of $\phi(t)$, we obtain that the number of possible $\phi$ 's is finite.

Consequently, it is enough to show that if $\phi$ is fixed, then those differential operators $D$ for which $\phi \circ D \in S_{1}$ constitute a finite dimensional space over $\mathbb{C}$.

Fix $\phi$, and let $D=\sum_{k=0}^{s} c_{k} \frac{\partial^{k}}{\partial t^{k}}$ be a differential operator such that $c_{s} \neq 0$ and $\phi \circ D \in S_{1}$. We prove that $s \leq m$. Since $\phi \circ D$ is a solution, we have

$$
\sum_{j=0}^{m} A_{j} \sum_{k=0}^{s} d_{k} \cdot \phi\left(\frac{\partial^{k}}{\partial t^{k}}\left(t^{j} x\right)\right)=0
$$

for every $x \in \mathbb{Q}(t)$, where $d_{k}=\phi\left(c_{k}\right)$. Since

$$
\frac{\partial^{k}}{\partial t^{k}}\left(t^{j} x\right)=\sum_{i=0}^{k}\left(\begin{array}{c}
k \\
i
\end{array}\right) \cdot \frac{\partial^{k-i}}{\partial t^{k-i}}\left(t^{j}\right) \cdot \frac{\partial^{i}}{\partial t^{i}} x
$$

we obtain

$$
\sum_{i=0}^{s} B_{i} \cdot \phi\left(\frac{\partial^{i}}{\partial t^{i}} x\right)=0
$$


where

$$
\begin{aligned}
B_{i} & =\sum_{j=0}^{m} \sum_{k=i}^{s} A_{j} \cdot\left(\begin{array}{c}
k \\
i
\end{array}\right) \cdot d_{k} \cdot \phi\left(\frac{\partial^{k-i}}{\partial t^{k-i}} t^{j}\right)= \\
& =\sum_{j=0}^{m} \sum_{\nu=0}^{s-i}\left(\begin{array}{c}
\nu+i \\
i
\end{array}\right) \cdot d_{\nu+i} \cdot A_{j} \cdot \phi\left(\frac{\partial^{\nu}}{\partial t^{\nu}} t^{j}\right)= \\
& =\sum_{\nu=0}^{s-i}\left(\begin{array}{c}
\nu+i \\
i
\end{array}\right) \cdot d_{\nu+i} \cdot \Gamma_{\nu} ;
\end{aligned}
$$

here we used the notation

$$
\Gamma_{\nu}=\sum_{j=0}^{m} A_{j} \cdot \phi\left(\frac{\partial^{\nu}}{\partial t^{\nu}} t^{j}\right) .
$$

Applying (22) with $x=1$ we obtain $B_{0}=0$. Then putting $x=t$ into (22) we obtain $B_{1}=0$. We continue, by substituting $t^{2}, t^{3}, \ldots$ into (22), and find that $B_{i}=0$ for every $i=0, \ldots, s$.

Now the equation $B_{s}=0$ gives $\Gamma_{0}=0$ by $c_{s} \neq 0$. Then, from $B_{s-1}=0$ we obtain $\Gamma_{1}=0$. Continuing this way we find that $\Gamma_{\nu}=0$ for every $\nu=0, \ldots, s$.

It is easy to check that

$$
\phi\left(\frac{\partial^{\nu}}{\partial t^{\nu}} t^{j}\right)=\left(\frac{\partial^{\nu}}{\partial X^{\nu}} X^{j}\right)_{X=\phi(t)}
$$

for every $\nu, j=0,1, \ldots$. Therefore, by $(24), \Gamma_{\nu}=0$ gives

$$
\begin{aligned}
0 & =\Gamma_{\nu}=\sum_{j=0}^{m} A_{j} \cdot \phi\left(\frac{\partial^{\nu}}{\partial t^{\nu}} t^{j}\right)= \\
& =\sum_{j=0}^{m} A_{j} \cdot\left(\frac{\partial^{\nu}}{\partial X^{\nu}} X^{j}\right)_{X=\phi(t)}= \\
& =\frac{\partial^{\nu}}{\partial X^{\nu}}\left(\sum_{j=0}^{m} A_{j} X^{j}\right)_{X=\phi(t)}= \\
& =P^{(\nu)}(\phi(t)) .
\end{aligned}
$$

Since this is true for every $\nu=0, \ldots, s$, we obtain that $\phi(t)$ is a root of $P$ of multiplicity at least $s$. However, $P$ is a nonzero polynomial of degree at most $m$, which gives $s \leq m$. 
We have proved that if $D$ is a differential operator on $\mathbb{Q}(t)$ such that $\phi \circ D$ is a solution of $(15)$ on $\mathbb{Q}(t)$, then the degree of $D$ is at most $m$. This implies that the set of these functions $\phi \circ D$ generate a linear space of finite dimension, which completes the proof.

Suppose that $a_{1}, \ldots, a_{n}$ are algebraic and $b_{i}, c_{i}$ generate a purely transcendental field of transcendence degree 1 . Then it follows from Theorems 5.4 and 5.6 that if (15) has a not identically zero additive solution on $K$, then the equation is trivial.

The following result generalizes this observation.

Theorem 5.7. Suppose that $a_{1}, \ldots, a_{n}$ are algebraic and the field $K=$ $\mathbb{Q}\left(b_{1}, \ldots, b_{n}, c_{1}, \ldots, c_{n}\right)$ is purely transcendental. If (15) has a not identically zero additive solution on $K$, then the equation is trivial.

Proof. Let $K=\mathbb{Q}\left(t_{1}, \ldots, t_{k}\right)$, where $t_{1}, \ldots, t_{k}$ are algebraically independent over $\mathbb{Q}$. Applying the argument of the proof of Theorem 5.6, it is enough to prove the following statement. Consider the equation

$$
\sum_{i_{1} \ldots i_{k}=0}^{m} A_{i_{1} \ldots i_{k}} \cdot f\left(t_{1}^{i_{1}} \ldots t_{k}^{i_{k}} \cdot x\right)=0
$$

where the coefficients $A_{i_{1} \ldots i_{k}}$ are algebraic. If (25) has an additive solution on $K$ which is not identically zero, then the equation is trivial.

It is easy to check that every additive solution of (25) defined on $K$ can be extended to $\mathbb{C}$ as an additive solution on $\mathbb{C}$. Therefore, if (25) has an additive solution on $K$ which is not identically zero, then there is such a solution on $\mathbb{C}$. By [3, Theorem 2.3] (or, by (iii) of Theorem 5.1), it follows that there is an automorphism $\phi$ of $\mathbb{C}$ which is a solution. This means that

$$
\sum_{i_{1} \ldots i_{k}=0}^{m} A_{i_{1} \ldots i_{k}} \cdot\left(\phi\left(t_{1}\right)\right)^{i_{1}} \ldots\left(\phi\left(t_{k}\right)\right)^{i_{k}}=0 .
$$

Since $\phi\left(t_{1}\right), \ldots, \phi\left(t_{k}\right)$ are algebraically independent over $\mathbb{Q}$ and the numbers $A_{i_{1} \ldots i_{k}}$ are algebraic, it follows that each $A_{i_{1} \ldots i_{k}}$ equals zero. Then the equation (25) is obviously trivial.

\section{Spectral synthesis of higher order}

In this section our aim is to prove the higher order analogue of Theorem 4.2. We shall need the following notation. 
If $\phi_{1}, \ldots, \phi_{k}$ are automorphisms of $\mathbb{C}$, then $\mathcal{A}_{\left(\phi_{1}, \ldots, \phi_{k}\right)}$ denotes the set of those functions which are finite sums of functions of the form

$$
\left(x_{1}, \ldots, x_{k}\right) \mapsto \prod_{i=1}^{k}\left(\phi_{i} \circ D_{i}\right)\left(x_{i}\right) \quad\left(x_{1}, \ldots, x_{n} \in K\right),
$$

where $D_{1}, \ldots, D_{k}$ are differential operators on $K$. By $\mathcal{A}_{\emptyset}$ we mean the class of constant functions.

The set of $k$-additive functions defined on the additive group $K^{k}$ is denoted by $V_{k}$.

Recall that $K^{*}$ denotes the Abelian group $\{x \in K: x \neq 0\}$ with respect to multiplication. The Abelian group $\underbrace{K^{*} \times \ldots \times K^{*}}_{k}$ will be denoted by $\left(K^{*}\right)^{k}$, where the operation is multiplication in every coordinate of the vectors of $\left(K^{*}\right)^{k}$. We put $V_{k}^{*}=\left\{\left.F\right|_{\left(K^{*}\right)^{k}}: F \in V_{k}\right\}$.

Lemma 6.1. $V_{k}^{*}$ is a variety on $\left(K^{*}\right)^{k}$.

Proof. It is clear that $V_{k}^{*}$ is a linear space over $\mathbb{C}$. Translation invariance follows from the fact that if $F: K^{k} \rightarrow \mathbb{C}$ is $k$-additive, then so is

$$
\left(x_{1}, \ldots, x_{k}\right) \mapsto F\left(c_{1} x_{1}, \ldots, c_{k} x_{k}\right) \quad\left(\left(x_{1}, \ldots, x_{k}\right) \in K^{k}\right)
$$

for every $\left(c_{1}, \ldots, c_{k}\right) \in\left(K^{*}\right)^{k}$. The proof of the statement that $V_{k}^{*}$ is closed is left to the reader (cf. [2]).

Lemma 6.2. Suppose that $m \in V_{k}$ and $\left.m\right|_{\left(K^{*}\right)^{k}}$ is an exponential; i.e. $m$ is nonzero on $\left(K^{*}\right)^{k}$, and $m(x y)=m(x) m(y)$ for every $x, y \in$ $\left(K^{*}\right)^{k}$. Then there are injective field homomorphisms $m_{1}, \ldots, m_{k}$ from $K$ into $\mathbb{C}$ such that

$$
m(x)=m\left(x_{1}, \ldots, x_{k}\right)=m_{1}\left(x_{1}\right) \cdots m_{k}\left(x_{k}\right) \quad\left(x_{1}, \ldots, x_{k} \in K\right) .
$$

Proof. By the multiplicativity of $m$,

$m\left(x_{1}, \ldots, x_{k}\right)=m\left(x_{1}, 1, \ldots, 1\right) \cdot m\left(1, x_{2}, 1, \ldots, 1\right) \cdot \ldots \cdot m\left(1, \ldots, 1, x_{k}\right)$.

Since $x_{i} \mapsto m\left(1, \ldots, 1, x_{i}, 1, \ldots, 1\right)$ is additive on $K$ and exponential on $K^{*}$, it is an injective homomorphism, which we denote by $m_{i}$. 
Theorem 6.3. Suppose that the transcendence degree of the field $K$ over $\mathbb{Q}$ is finite. Let $F: K^{k} \rightarrow \mathbb{C}$ be a $k$-additive function. Let $m_{1}, \ldots, m_{k}$ be injective homomorphisms of $K$, let

$$
m(x)=m\left(x_{1}, \ldots, x_{k}\right)=m_{1}\left(x_{1}\right) \cdots m_{k}\left(x_{k}\right)
$$

for every $x_{1}, \ldots, x_{k} \in K$, and let $\phi_{i}$ be an extension of $m_{i}$ to $\mathbb{C}$ as an automorphism of $\mathbb{C}$ for every $i=1, \ldots, k$. Then the following are equivalent.

(i) $F=p \cdot m$ on $\left(K^{*}\right)^{k}$, where $p$ is a local polynomial on $\left(K^{*}\right)^{k}$.

(ii) $F=p \cdot m$ on $\left(K^{*}\right)^{k}$, where $p$ is a generalized polynomial on $\left(K^{*}\right)^{k}$.

(iii) $F=p \cdot m$ on $\left(K^{*}\right)^{k}$, where $p$ is a polynomial on $\left(K^{*}\right)^{k}$.

(iv) $F \in \mathcal{A}_{\left(\phi_{1}, \ldots, \phi_{k}\right)}$.

Proof. The implications (iii) $\Longrightarrow($ ii $) \Longrightarrow$ (i) are obvious.

(i) $\Longrightarrow$ (iv): We prove by induction on $k$. The case $k=1$ is covered by Theorem 4.2. Let $k>1$, and suppose the statement is true for $k-1$. In the proof of the induction step we shall assume that $k=2$. It is easy to check that the same argument works in the general case.

Suppose $F$ is biadditive on $K^{2}$, and $F=p \cdot m$ on $K^{2}$, where $p$ is a local polynomial on $\left(K^{*}\right)^{2}$, and

$$
m(x, y)=m_{1}(x) \cdot m_{2}(y)
$$

for every $x, y \in K$, where $m_{1}$ and $m_{2}$ are given injective homomorphisms of $K$. Let $\phi$ be an extension of $m_{1}$ and $\psi$ be an extension of $m_{2}$ to $\mathbb{C}$ as automorphisms.

Let $T$ be a maximal subset of $K$ consisting of algebraically independent elements over $\mathbb{Q}$. Then $T$ is finite; let $T=\left\{t_{1}, \ldots, t_{N}\right\}$. Let $G$ denote the subgroup of $K^{*}$ generated by $T$. Then $G^{2}$ is a finitely generated subgroup of $\left(K^{*}\right)^{2}$, and thus $p$ is a polynomial on $G^{2}$. Therefore, $p$ is a finite sum of terms of the form $a_{1} \cdots a_{s}$, where each factor $a_{i}$ is either additive on $\left(K^{*}\right)^{2}$ or is a constant. Note that the additivity of the function $a:\left(K^{*}\right)^{2} \rightarrow \mathbb{C}$ means

$$
a(x y)=a(x)+a(y) \quad\left(x, y \in\left(K^{*}\right)^{2}\right) .
$$

Let $x=t_{1}^{j_{1}} \cdots t_{N}^{j_{N}}, y=t_{1}^{k_{1}} \cdots t_{N}^{k_{N}}$ be arbitrary elements of $G$, where $j_{1}, \ldots, j_{N}, k_{1}, \ldots, k_{N} \in \mathbb{Z}$. Let $a:\left(K^{*}\right)^{2} \rightarrow \mathbb{C}$ be additive. If $a\left(t_{i}, 1\right)=$ $\alpha_{i}$ and $a\left(1, t_{i}\right)=\beta_{i}$, then

$$
a(x, y)=\alpha_{1} j_{1}+\ldots+\alpha_{N} j_{N}+\beta_{1} k_{1}+\ldots+\beta_{N} k_{N}
$$


and

$$
m(x, y)=\phi\left(t_{1}\right)^{j_{1}} \cdots \phi\left(t_{N}\right)^{j_{N}} \cdot \psi\left(t_{1}\right)^{k_{1}} \cdots \psi\left(t_{N}\right)^{k_{N}}
$$

for every $j_{1}, \ldots, j_{N}, k_{1}, \ldots, k_{N} \in \mathbb{Z}$. Therefore, the value of the function $a_{1} \cdots a_{s} \cdot m$ at the point $(x, y)$ is a linear combination, with complex coefficients, of terms of the form

$$
\begin{array}{r}
j_{1}^{c_{1}} \cdots j_{N}^{c_{N}} \cdot k_{1}^{d_{1}} \cdots k_{N}^{d_{N}} \cdot \phi\left(t_{1}\right)^{j_{1}} \cdots \phi\left(t_{N}\right)^{j_{N}} \cdot \psi\left(t_{1}\right)^{k_{1}} \cdots \psi\left(t_{N}\right)^{k_{N}}= \\
=\phi\left(j_{1}^{c_{1}} \cdots j_{N}^{c_{N}} \cdot t_{1}^{j_{1}} \cdots t_{N}^{j_{N}}\right) \cdot \psi\left(k_{1}^{d_{1}} \cdots k_{N}^{d_{N}} \cdot t_{1}^{k_{1}} \cdots t_{N}^{k_{N}}\right),
\end{array}
$$

where $c_{i}, d_{i}$ are nonnegative integers. Then the value of $p \cdot m$ at the point $(x, y)$ is also a linear combination of terms of the same form.

It is easy to see that for every choice of the nonnegative integers $c_{i}, d_{i}$ there are differential operators $D$ and $E$ on $\mathbb{Q}(T)$ such that

$$
D\left(t_{1}^{j_{1}} \cdots t_{N}^{j_{N}}\right)=j_{1}^{c_{1}} \cdots j_{N}^{c_{N}} \cdot t_{1}^{j_{1}} \cdots t_{N}^{j_{N}}
$$

and

$$
E\left(t_{1}^{k_{1}} \cdots t_{N}^{k_{N}}\right)=k_{1}^{d_{1}} \cdots k_{N}^{d_{N}} \cdot t_{1}^{k_{1}} \cdots t_{N}^{k_{N}}
$$

for every $j_{1}, \ldots, j_{N}, k_{1}, \ldots, k_{N} \in \mathbb{Z}$ (see the proof of Theorem 3.4). Now it follows from (27) that the map $p \cdot m$, restricted to $G^{2}$, is the finite sum of the form

$$
(\phi \circ D)(x) \cdot(\psi \circ E)(y) \quad(x, y \in G),
$$

where $D$ and $E$ are differential operators. Let

$$
(p \cdot m)(x, y)=\sum_{\nu=1}^{S}\left(\phi \circ D_{\nu}\right)(x) \cdot\left(\psi \circ E_{\nu}\right)(y)
$$

for every $x, y \in G$. The maps $D_{\nu}, E_{\nu}$ can be extended to $K$ as differential operators. Then the extended maps (denoted by the same letter) are additive on $K$ and $D_{\nu} / j, E_{\nu} / j$ are polynomials on $K^{*}$. The extended differential operators make the right hand side of (28) welldefined on $\left(K^{*}\right)^{2}$. We prove that $(28)$ holds everywhere on $\left(K^{*}\right)^{2}$.

Let $x \in G$ be fixed. Then the left hand side of (28) equals $q(y)$. $m_{2}(y)$, where $q(y)=p(x, y) \cdot m_{1}(x)$ for every $y \in K$. It is easy to check that the function $y \mapsto p(x, y)$ is a local polynomial on $K$, and thus so is $q$. 
Let $\left(\phi \circ D_{\nu}\right)(x)=\gamma_{\nu}$ and $\psi^{-1}\left(\gamma_{\nu}\right)=\delta_{\nu}$. Then the right hand side of (28) equals $\psi \circ E$, where $E=\sum_{\nu=1}^{S} \delta_{\nu} E_{\nu}$ is a differential operator on $K$. By (28),

$$
q(y) \cdot m_{2}(y)=(\psi \circ E)(y)
$$

on $G$. By the equivalence of the statements (i) and (iv) of Theorem 4.2 , there is a unique differential operator $\bar{E}$ on $K$ such that

$$
q(y) \cdot m_{2}(y)=(\psi \circ \bar{E})(y)
$$

for every $y \in K$. Then $\psi \circ E=\psi \circ \bar{E}$ on $G$, since both sides equal $q \cdot m_{2}$ on $G$. Since $\psi$ is injective, this implies $E=\bar{E}$ on $G$.

Since $E$ and $\bar{E}$ are differential operators on $K$, they are additive on $K$, and $E / j$ and $\bar{E} / j$ are polynomials on $K^{*}$ by definition. Since $E=\bar{E}$ on $G$, it follows from Lemma 3.6 that $E=\bar{E}$ on $K$, and thus (28) holds for every $y \in K$.

Now let $y \in K$ be fixed. Repeating the argument above we can see that (28) holds for every $x \in K$. Therefore, we have $p \cdot m \in \mathcal{A}_{(\phi, \psi)}$. This proves the implication (i) $\Longrightarrow$ (iv).

(iv) $\Longrightarrow$ (iii): It is enough to show that the map $\left(x_{1}, \ldots, x_{k}\right) \mapsto$ $\prod_{i=1}^{k}\left(\phi_{i} \circ D_{i}\right)\left(x_{i}\right)$ is of the form $p \cdot m$ on $K^{k}$. By the equivalence of the statements (iii) and (iv) of Theorem 4.2, there are polynomials $p_{i}$ on $K^{*}$ such that $\phi_{i} \circ D_{i}=p_{i} \cdot m_{i}$ on $K$. Then

$$
\prod_{i=1}^{k}\left(\phi_{i} \circ D_{i}\right)\left(x_{i}\right)=\prod_{i=1}^{k} p_{i}\left(x_{i}\right) \cdot m_{i}\left(x_{i}\right)=\left(\prod_{i=1}^{k} p_{i}\left(x_{i}\right)\right) \cdot m\left(x_{1}, \ldots, x_{k}\right)
$$

on $K$. It is clear that $\left(x_{1}, \ldots, x_{k}\right) \mapsto \prod_{i=1}^{k} p_{i}\left(x_{i}\right)$ is a polynomial on $K^{k}$, which completes the proof.

Remark 6.4. The proof of the implication (i) $\Longrightarrow$ (iv) gives the following: in the representation of $p \cdot m$ as a sum of functions of the form (26), the sum of the degrees of the differential operators equals the degree of $p$ in every term.

Theorem 6.5. Suppose that the transcendence degree of the field $K$ over $\mathbb{Q}$ is finite. Then spectral synthesis holds in every variety on $\left(K^{*}\right)^{k}$ consisting of $k$-additive functions (with respect to addition).

Proof. Since $K$ is countable, so is the Abelian group $\left(K^{*}\right)^{k}$. Let $V$ be a variety on $\left(K^{*}\right)^{k}$ consisting of $k$-additive functions. By Theorem 2.2 , local spectral synthesis holds on $K^{*}$, and thus $V$ is spanned by 
local polynomial-exponential functions. Since, by Theorem 6.3, every local polynomial-exponential function contained by $V$ is a polynomialexponential function, it follows that $V$ is spanned by polynomialexponential functions.

\section{The space of solutions of linear func- tional equations}

We continue the description of the solutions of

$$
\sum_{i=1}^{n} a_{i} f\left(b_{i} x+c_{i} y\right)=0
$$

where $a_{i}, b_{i}, c_{i}$ are given complex numbers and $f: \mathbb{C} \rightarrow \mathbb{C}$ is the unknown function. Let $K=\mathbb{Q}\left(b_{1}, \ldots, b_{n}, c_{1}, \ldots, c_{n}\right)$.

Our aim is to generalize Theorem 5.1 to the case of $k>1$. Let $S_{k}$ denote the set of those solutions of (29) defined on $K$ which are generalized monomials of degree $k$. In [3, Theorem 3.5] it was proved that if $S_{k}$ contains a nonzero function, then there are field automorphisms $\phi_{1}, \ldots, \phi_{k}$ of $\mathbb{C}$ such that $\phi_{1} \cdots \phi_{k} \in S_{k}$. The proof depends on the fact that spectral analysis holds in a certain variety. Our proof of Theorem 7.3 is based on the observation that, by Theorem 6.5, spectral synthesis holds in the same variety.

Let $M_{k}$ denote the set of the functions $F: K^{k} \rightarrow \mathbb{C}$ such that $F$ is $k$-additive, and the function $x \mapsto F\left(s_{1} x, s_{2} x, \ldots, s_{k} x\right)$ is a solution of (29) on $K$ for every $s_{1}, s_{2}, \ldots s_{k} \in K^{*}$. We put

$$
M_{k}^{*}=\left\{\left.F\right|_{\left(K^{*}\right)^{k}}: F \in M_{k}\right\} .
$$

Lemma 7.1. $M_{k}^{*}$ is a variety on $\left(K^{*}\right)^{k}$.

Proof. It is easy to see that $M_{k}$ is a closed linear space over $\mathbb{C}$, and then so is $M_{k}^{*}$. Translation invariance means that if $F \in M_{k}^{*}$, then the map $\left(x_{1}, \ldots, x_{k}\right) \mapsto F\left(c_{1} x_{1}, \ldots, c_{k} x_{k}\right)\left(x_{1}, \ldots, x_{k} \in K^{*}\right)$ also belongs to $M_{k}^{*}$ for every $c_{1}, \ldots, c_{K} \in K^{*}$, which is easily seen from the definition of $M_{k}^{*}$.

The diagonal of the function $F: K^{k} \rightarrow \mathbb{C}$ is defined as $f(x)=$ $F(x, \ldots, x)(x \in K)$, and is denoted by $\operatorname{diag} F$. 


\section{Lemma 7.2.}

$$
S_{k}=\left\{\operatorname{diag} F: F \in M_{k}\right\} .
$$

Proof. It is clear that $\operatorname{diag} F \in S_{k}$ for every $F \in M_{k}$. We prove the converse. Let $f: K \rightarrow \mathbb{C}$ be an element of $S_{k}$. Then $f$ is a solution (29) on $K$, and $f=\operatorname{diag} F$ for a symmetric $k$-additive function $F: K^{k} \rightarrow$ $\mathbb{C}$. We prove $F \in M_{k}$. We have to show that for every $\left(s_{1}, \ldots, s_{k}\right) \in$ $\left(K^{*}\right)^{k}$ the diagonal of the function

$$
G\left(x_{1}, \ldots, x_{k}\right)=F\left(s_{1} x_{1}, \ldots, s_{k} x_{k}\right)
$$

belongs to $S_{k}$. Let $\operatorname{diag} G=g$. Then, by (6) we have

$$
g(x)=F\left(s_{1} x, \ldots, s_{k} x\right)=\frac{1}{k !} \cdot \Delta_{s_{1} x} \Delta_{s_{2} x} \ldots \Delta_{s_{k} x} f(0)=\sum_{j=1}^{M} \pm f\left(e_{j} x\right)
$$

with suitable $e_{1}, \ldots, e_{M} \in K$. Since $x \mapsto f(e x)$ belongs to $S_{k}$ for every $e \in K$, it follows that $g \in S_{k}$, and thus $F \in M_{k}$.

Theorem 7.3. (i) For every function $F \in M_{k}, F$ is an exponential monomial on $\left(K^{*}\right)^{k}$ if and only if $F \in \mathcal{A}_{\left(\phi_{1}, \ldots, \phi_{k}\right)}$, where $\phi_{1}, \ldots, \phi_{k}$ are automorphisms of $\mathbb{C}$ and $\prod_{i=1}^{k} \phi_{i}(x)$ is a solution of (29).

(ii) The variety $M_{k}^{*}$ is spanned by the classes $M_{k}^{*} \cap \mathcal{A}_{\left(\phi_{1}, \ldots, \phi_{k}\right)}$, where $\phi_{1}, \ldots, \phi_{k}$ are as above.

Proof. (i) Suppose that $F \in M_{k}$ is an exponential monomial on $\left(K^{*}\right)^{k}$, and let $F=p \cdot m$, where $p$ is a polynomial, and $m$ is an exponential on $\left(K^{*}\right)^{k}$. Since $M_{k}^{*}$ is a variety, $p \cdot m \in M_{k}^{*}$ implies $m \in M_{k}^{*}$ by Lemma 2.1. Note that $M_{k} \subset V_{k}$ and $M_{k}^{*} \subset V_{k}^{*}$. Therefore, by Lemma 6.2 , there are injective field homomorphisms $m_{1}, \ldots, m_{k}$ from $K$ into $\mathbb{C}$ such that

$$
m\left(x_{1}, \ldots, x_{k}\right)=m_{1}\left(x_{1}\right) \cdots m_{k}\left(x_{k}\right) \quad\left(x_{1}, \ldots, x_{k} \in K^{*}\right) .
$$

Let $\phi_{i}$ be an extension of $m_{i}$ to $\mathbb{C}$ as an automorphism of $\mathbb{C}$. Then $m(x, \ldots, x)=\prod_{i=1}^{k} \phi_{i}(x)$ is a solution of $(29)$ on $K$. The rest of the statement (i) follows from Theorems 6.3.

Statement (ii) is a consequence of Theorem 6.5.

The statement of the next theorem follows immediately from Lemma 7.2 and (ii) of Theorem 7.3. 
Theorem 7.4. The set $S_{k}$ is spanned by the classes $S_{k} \cap \mathcal{A}_{\left(\phi_{1}, \ldots, \phi_{k}\right)}$, where $\phi_{1}, \ldots, \phi_{k}$ are automorphisms of $\mathbb{C}$, and $\prod_{i=1}^{k} \phi_{i}$ is a solution of (29).

Let $S_{\leq k}$ denote the set of those solutions of (29) defined on $K$ which are generalized polynomials of degree at most $k$. Then $S_{\leq k}$ is a closed linear space over $\mathbb{C}$.

Theorem 7.5. The set $S_{\leq k}$ is spanned by the classes $S_{m} \cap \mathcal{A}_{\left(\phi_{1}, \ldots, \phi_{m}\right)}$, where $0 \leq m \leq k, \phi_{1}, \ldots, \phi_{m}$ are automorphisms of $\mathbb{C}$, and $\prod_{i=1}^{m} \phi_{i}$ is a solution of (29).

Proof. Let $f \in S_{\leq k}$ be arbitrary. Then $f=\sum_{m=0}^{k} f_{m}$, where $f_{m}$ is a generalized monomial of degree $m$ for every $m=1, \ldots, k$, and $f_{0}$ is constant. By [3, Lemma 2.1], each of the functions $f_{0}, \ldots, f_{k}$ is a solution of (29). Therefore, we have $f_{m} \in S_{m}$ for every $m=1, \ldots, k$.

It is enough to show that each $f_{m}$ is in the closure of the linear space spanned by the classes $\mathcal{A}_{\left(\phi_{1}, \ldots, \phi_{m}\right)} \cap S_{m}$, where $\phi_{1}, \ldots, \phi_{m}$ are automorphisms of $\mathbb{C}$, and $\prod_{i=1}^{m} \phi_{i}(x) \in S_{m}$. If $m \geq 1$ then this is true by Theorem 7.4 .

If $m=0$, then there are two cases to consider. If $\sum_{i=1}^{n} a_{i} \neq 0$, then the only constant solution of (29) is the zero function, so $f_{0}=0$. On the other hand, if $\sum_{i=1}^{n} a_{i}=0$, then all constant functions are solutions of (29). Then the statement is true, since $\mathcal{A}_{\emptyset}$ is the class of constant functions.

Corollary 7.6. Suppose that the equation (29) is normal of degree $k$. Then the linear space of its solutions defined on $K$ is spanned by the classes $S_{m} \cap \mathcal{A}_{\left(\phi_{1}, \ldots, \phi_{m}\right)}$, where $0 \leq m \leq k, \phi_{1}, \ldots, \phi_{m}$ are automorphisms of $\mathbb{C}$, and $\prod_{i=1}^{m} \phi_{i}$ is a solution of (29).

In particular, this is true for the equations (3) and (4) with $k=$ $n-2$.

Theorem 7.7. Let $S$ denote the set of solutions of (29) defined on $K$.

(i) If the equation is normal, then the set of polynomials on the additive group of $K$ is dense in $S$.

(ii) If the equation is normal and $\sum_{i=1}^{n} a_{i}=0$, then spectral synthesis holds in $S$.

(iii) If the equation is normal and translation invariant, then spectral synthesis holds in $S$. 
Proof. If $\phi$ is an automorphism of $\mathbb{C}$, and $D$ is a differential operator on $K$, then the function $\phi \circ D$ is additive on $K$. Therefore, each class $\mathcal{A}_{\left(\phi_{1}, \ldots, \phi_{k}\right)}$ consists of polynomials. Thus (i) follows from Corollary 7.6.

Let 1 denote the identically 1 function defined on $K$. If $\sum_{i=1}^{n} a_{i}=$ 0 , then $\mathbf{1}$ is a solution. Since $\mathbf{1}$ is an exponential function, every polynomial in $S$ is, in fact, a polynomial-exponential function. Thus (ii) follows from (i).

Suppose that the equation is normal and translation invariant. If $S=\{0\}$, then spectral synthesis holds in $S$. If $S$ contains a nonzero generalized polynomial $p$, then it follows from the translation invariance of $S$ that $\Delta_{h_{1}} \ldots \Delta_{h_{k}} p \in S$ for every $k$ and $h_{1}, \ldots, h_{k} \in \mathbb{C}$. If $k=\operatorname{deg} p$, then $\Delta_{h_{1}} \ldots \Delta_{h_{k}} p$ is a nonzero constant for suitable $h_{1}, \ldots, h_{k}$. Thus $\mathbf{1} \in S$, and thus (iii) follows from (ii).

\section{An example and concluding remarks}

The following example serves as an illustration: it shows how to use our previous results in order to determine the set of solutions of a given equation. We consider the equation

$$
\begin{aligned}
-2 f(y) & +f(t x+y)+f((1-t) x+y)+ \\
& +f\left(\left(t^{2}-t\right) x+y\right)-f\left(\left(t^{2}-t+1\right) x+y\right)=0,
\end{aligned}
$$

where $t$ is a fixed transcendental number. The equation is of the form (3) where $n=5,\left(a_{1}, \ldots, a_{5}\right)=(-2,1,1,1,-1)$ and $\left(b_{1}, \ldots, b_{5}\right)=$ $\left(0, t, 1-t, t^{2}-t, t^{2}-t+1\right)$. Thus every solution is a generalized polynomial of degree at most three.

In order to simplify notation, we shall write $x^{\prime}$ instead of $\frac{\partial}{\partial t} x, x^{\prime \prime}$ instead of $\frac{\partial^{2}}{\partial t^{2}} x$ etc. It is easy to check that we have

$$
\sum_{i=1}^{5} a_{i} \cdot b_{i}^{n}=0(n=0,1,2), \quad \sum_{i=1}^{5} a_{i} \cdot b_{i}^{3} \neq 0 .
$$

We shall also need

$$
\sum_{i=1}^{5} a_{i} \cdot b_{i}^{(n)} \cdot b_{i}^{(m)}=\left\{\begin{aligned}
2 & \text { if } n=m=1 \\
-2 & \text { if } n=2, m=0 \\
0 & \text { if } n \geq m \geq 0, n+m \neq 2 .
\end{aligned}\right.
$$

It is easy to see that the equation is trivial; that is, every additive function is a solution. (Since $a_{1}, \ldots, a_{5}$ are integers and $K=\mathbb{Q}(t)$ is 
purely transcendental, it follows from Theorem 5.7 that if (30) were not trivial, it wouldn't have any nonzero solution on $K$.) Since the maps $x \mapsto x^{(n)}$ are additive functions, we have

$$
\sum_{i=1}^{5} a_{i} \cdot b_{i}^{(n)}=0 \quad(n=1,2, \ldots) .
$$

Let $\phi, \psi$ be automorphisms of $\mathbb{C}$, and suppose that $g=\phi \cdot \psi$ is a solution of (30). If we substitute $f=g, y=0$ and $x=1$ into (30), the left hand side of the equality obtained equals $\phi(t)^{2}+\psi(t)^{2}-$ $2 \phi(t) \psi(t)=(\phi(t)-\psi(t))^{2}$. Since $g$ is a solution, we get $\phi(t)=\psi(t)$. This implies that $\phi=\psi$ on $K$.

We prove that there is no nonzero solution which is a generalized monomial of degree 3. Indeed, suppose there is such a solution. Then it follows from Theorem 7.4 that there are automorphisms $\phi, \psi, \chi$ of $\mathbb{C}$ such that $\phi \cdot \psi \cdot \chi$ is a solution. Then, by [3, Theorem 3.8], the functions $\phi \cdot \psi, \phi \cdot \chi, \psi \cdot \chi$ are also solutions. As we saw above, this implies that $\phi=\psi=\chi$ on $K$, and thus $\phi^{3}$ is a solution. Then we have

$$
0=\sum_{i=1}^{5} a_{i} \cdot \phi\left(b_{i}\right)^{3}=\phi\left(\sum_{i=1}^{5} a_{i} \cdot b_{i}^{3}\right),
$$

which contradicts (31). This proves that there is no nonzero solution which is a generalized monomial of degree 3 .

Therefore, in order to determine all solutions of (30), it is enough to describe the set $S_{2}$ of those solutions which are defined on $K$ and are generalized monomials of degree two.

If $\phi, \psi$ are automorphisms of $\mathbb{C}$, then $\mathcal{A}_{(\phi, \psi)}$ denotes the set of functions of the form $\sum_{j=1}^{N}\left(\phi \circ D_{j}\right) \cdot\left(\psi \circ E_{j}\right)$, where $D_{j}$ and $E_{j}$ are differential operators on $K$. By Theorem 7.4, the set $S_{2}$ is spanned by $S_{2} \cap \mathcal{A}_{(\phi, \psi)}$, where $\phi, \psi$ are automorphisms of $\mathbb{C}$ such that $\phi \cdot \psi$ is a solution of (30). Since this implies $\phi=\psi$, we may confine our attention to the sets $\mathcal{A}_{(\phi, \phi)}$. It is clear that

$$
\mathcal{A}_{(\phi, \phi)}=\phi\left(\mathcal{A}_{(j, j)}\right)
$$

for every automorphism $\phi$, where $j$ denotes the identity map. Also, $f$ is a solution of (7) if and only if $\phi \circ f$ is, so we only need to describe $S_{2} \cap \mathcal{A}_{(j, j)}$.

Since every differential operator on $K=\mathbb{Q}(t)$ is the linear combination of the maps $x \mapsto x^{(n)}(x \in K, n=0,1, \ldots)$, the elements of 
$\mathcal{A}_{(j, j)}$ are linear combinations of the maps

$$
f_{n, m}(x)=x^{(n)} \cdot x^{(m)} \quad(x \in K, 0 \leq m \leq n) .
$$

In order to determine which linear combinations of the maps $f_{n, m}$ are solutions, we have to compute the sums

$$
S_{n, m}(x, y)=\sum_{i=1}^{5} a_{i} \cdot f_{n, m}\left(b_{i} x+y\right),
$$

and determine those linear combinations of the functions $S_{n, m}$ which are identically zero on $K=\mathbb{Q}(t)$. A computation, based on (32) and (33), shows that we have $S_{0,0}=S_{1,0}=S_{1,1}+S_{2,0}=0$ on $K$, and if a linear combinations of the functions $S_{n, m}$ is zero on $K$, then it is also a linear combination of $S_{0,0}, S_{1,0}$ and $S_{1,1}+S_{2,0}$. We omit the details. This means that $S_{2} \cap \mathcal{A}_{(j, j)}$ is the linear span of the functions $x^{2}, x \cdot x^{\prime}$ and $\left(x^{\prime}\right)^{2}+x \cdot x^{\prime \prime}$. Summing up:

The space of solutions of (30) defined on $K$ is the closed linear hull of all additive functions and the functions

$$
\phi^{2}, \phi \cdot\left(\phi \circ \frac{\partial}{\partial t}\right) \text { and }\left(\phi \circ \frac{\partial}{\partial t}\right)^{2}+\phi \cdot\left(\phi \circ \frac{\partial^{2}}{\partial t^{2}}\right),
$$

where $\phi$ is an arbitrary injective homomorphism of $K$.

The example above shows that some of the results of Section 5 cannot be generalized for solutions of degree greater than 1 . Theorem 5.2 says that if $a_{1}, \ldots, a_{n}$ are algebraic numbers and the injective homomorphism $\phi$ is a solution, then $\phi \circ D$ is also a solution for every differential operator $D$. In the example above, $\phi \cdot \phi$ is a solution for every $\phi$, but $\left(\phi \circ \frac{\partial}{\partial t}\right)^{2}$ is not a solution, so the analogy is false for monomials of degree 2. This implies that the analogy of Corollary 7.6 is also false for monomials of degree 2 .

Theorem 5.6 says that if $b_{i}, c_{i} \in \mathbb{Q}(t)$, where $t$ is transcendental over $\mathbb{Q}$, then the equation is either trivial or $S_{1}$ is of finite dimension over $\mathbb{C}$. The analogous statement would be that if $b_{i}, c_{i} \in \mathbb{Q}(t)$, then either every monomial of degree two is a solution, or $S_{2}$ is of finite dimensional. The example above shows that this is not true in general. We can see that the analogue of Theorem 5.7 is also false in $S_{2}$.

We remark, however, that if the space of additive solutions of an equation (3) is of finite dimensional, then so is the space of those 
solutions which are generalized monomials of degree two. (This follows from the fact that if $A(x, y)$ is symmetric and biadditive, and $x \mapsto$ $A(x, x)$ is a solution, then the functions $y \mapsto A(x, y)(x \in K)$ are additive solutions. If these latter functions span a linear space of finite dimension over $\mathbb{C}$ generated by the additive functions $a_{1}, \ldots, a_{k}$, then $A(x, y)$ is the linear combination of the functions $a_{i}(x) \cdot a_{j}(y)$ $(i, j=1, \ldots, k)$.)

Although the description of the set of solutions of a given equation can be difficult, the example above shows that, at least in principle, the description is possible in the case of many equations.

We conclude with some remarks concerning the 'generic' or 'random' equation. By that we mean an equation (1) in which the numbers $a_{i}, b_{i}, c_{i}$ are algebraically independent over $\mathbb{Q}$. Such an equation is normal, but not translation invariant. An injective homomorphism $\phi$ is a solution if and only if

$$
\sum_{i=1}^{n} a_{i} \phi\left(b_{i}\right)=\sum_{i=1}^{n} a_{i} \phi\left(c_{i}\right)=0
$$

holds. This implies that the equations is not trivial (not every additive function is a solution), but $S_{1}$ is of infinite dimensional. One can prove that $S_{1}$ is spanned by the injective homomorphisms satisfying (34). Note that differential operators do not appear in the description of $S_{1}$. The description of higher order solutions is left to the reader. 


\section{References}

[1] J. A. Baker, A general functional equation and its stability, Proc. Am. Math. Soc. 133 (2004), no. 6, 1657-1664.

[2] G. Kiss, Linear functional equations with algebraic parameters, submitted.

[3] G. Kiss and A. Varga, Existence of nontrivial solutions of linear functional equations, to appear in Aequationes Math.

[4] M. Laczkovich, Polynomial mappings on Abelian groups, Aequationes Math. 68 (2004), 177-199.

[5] M. Laczkovich, Local spectral synthesis on Abelian groups, to appear in Acta Math. Hungar.

[6] M. Laczkovich and L. Székelyhidi, Spectral synthesis on discrete Abelien groups, Math. Proc. Camb. Phil. Soc. 143 (2007), 103120 .

[7] L. Székelyhidi, On a class of linear functional equations, Publ. Math. (Debrecen) 29 (1982), 19-28.

[8] L. Székelyhidi, The failure of spectral synthesis on some types of discrete Abelian groups, J. Math. Anal. and Applications 291 (2004), 757-763.

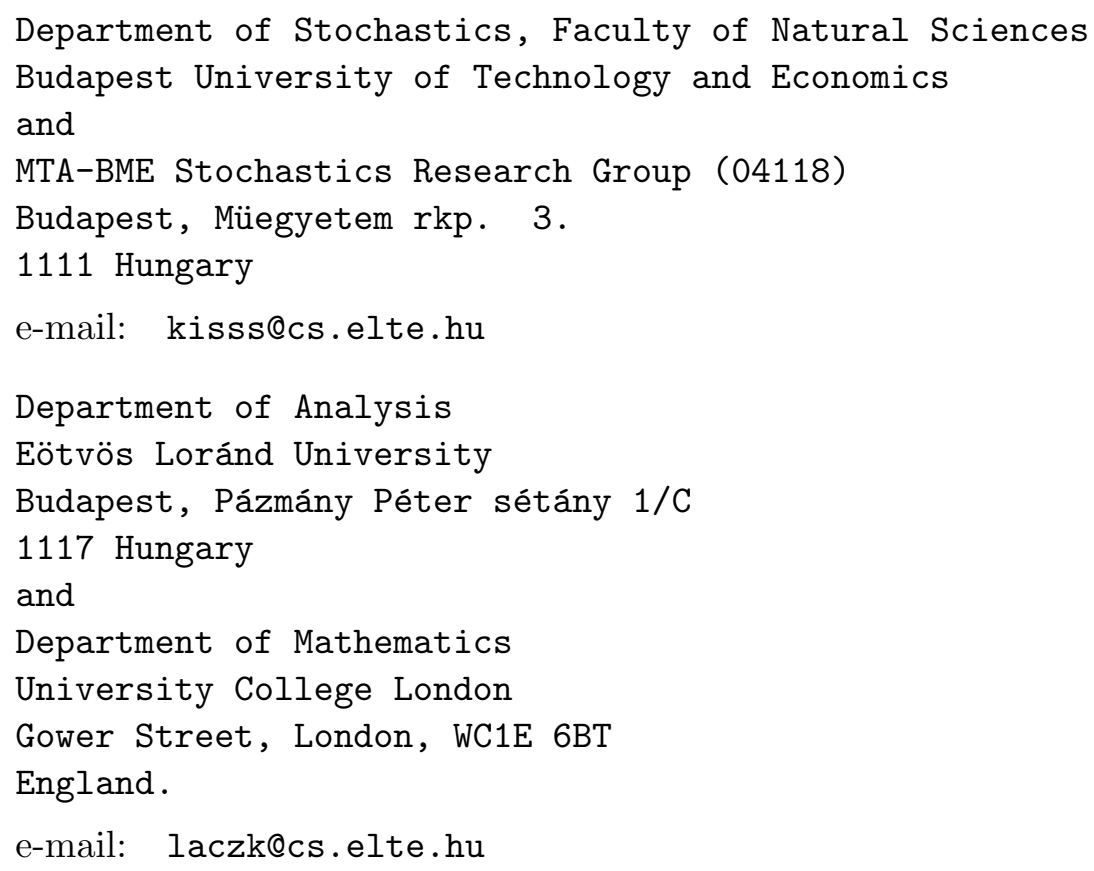

